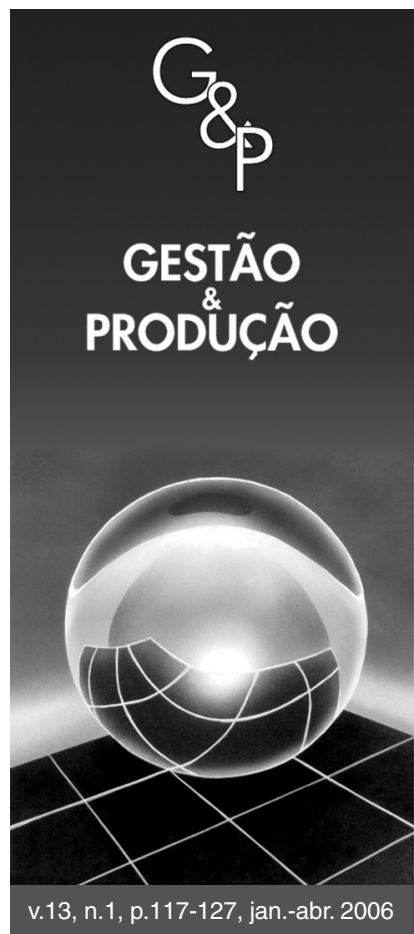

\title{
AVALIAÇÃO QUALITATIVA PARACONSISTENTE DO PROCESSO DE IMPLANTAÇÃO DE UM SISTEMA DE GESTÃO AMBIENTAL
}

\author{
Carlos Alberto Ferreira Bispo \\ Edson Walmir Cazarini \\ Departamento de Engenharia de Produção, \\ Escola de Engenharia de São Carlos, Universidade de São Paulo, \\ e-mail: cafbispo@sc.usp.br, cazarini@sc.usp.br
}

Resumo

O objetivo deste artigo é apresentar a proposta de um novo processo de avaliação qualitativa de implantação de um Sistema de Gestão Ambiental (SGA) elaborado com base na norma ISO 14001: 2004 e com a intenção de sua futura certificação por essa norma. A avaliação proposta aplica os recursos da Lógica Paraconsistente Anotada de Dois Valores (LPA2V). Apresenta-se como é feita essa avaliação, porque ela é diferente das demais avaliações qualitativas e porque é importante para a área de Gestão Ambiental. Faz-se uma comparação entre os resultados obtidos simuladamente com uma avaliação qualitativa tradicional e utilizando a avaliação qualitativa paraconsistente. Apresentam-se as ferramentas apropriadas para auxiliar a visualização do cenário que envolve análises paraconsistentes, ou seja, o Algoritmo Para-Analisador e o Quadrado Unitário do Plano Cartesiano.

Palavras-chave: sistema de gestão ambiental, avaliação qualitativa paraconsistente, lógica paraconsistente anotada de dois valores.

\section{Introdução}

A técnica que será empregada neste artigo utiliza os fundamentos de uma das Lógicas Paraconsistentes (Costa et al., 1991) para realizar uma avaliação qualitativa no processo de implantação de um Sistema de Gestão Ambiental, cujos passos, para essa implantação, são baseados na norma NBR ISO 14001: 2004. Esse embasamento visa sua futura certificação.

Sabe-se que aquela norma, assim como aquele sistema, já possui suas próprias formas de avaliação, baseados predominantemente em indicadores quantitativos. Porém, conforme ficará demonstrado no artigo, propõe-se uma forma alternativa de avaliação qualitativa baseada na Lógica Paraconsistente Anotada de Dois Valores, o que levará a resultados mais precisos e mais próximos do real cenário do sistema avaliado.

Como ainda não foi possível colocar em prática a técnica proposta, sua demonstração será feita por meio de duas simulações. A primeira utilizará a técnica da avalia- ção qualitativa tradicional, a segunda empregará a técnica da avaliação qualitativa paraconsistente. A seguir, serão feitas as devidas comparações entre os resultados obtidos pelas duas técnicas.

\section{Avaliação quantitativa vs. avaliação qualitativa}

A avaliação é o ato de atribuir um valor a um determinado "objeto", podendo resultar em um valor matemático, estatístico ou lógico, um preço, uma nota, um mérito, etc. O termo "objeto" está sendo empregado de forma genérica para representar tudo aquilo que pode ser avaliado de alguma forma.

Existem dois tipos de avaliação: a quantitativa e a qualitativa. A avaliação quantitativa é baseada em métodos empregados nas Ciências Exatas para produzir um ou mais resultados, proporcionando-lhes plena confiabilida- 
de. Por exemplo, na avaliação da situação financeira de uma empresa, se os dados forem fiéis e confiáveis, que leva a um cenário bem claro e específico de como andam as suas finanças, o resultado é inquestionável. Outros exemplos: a avaliação das vendas efetuadas devidamente estratificadas por categoria de produto, por região ou por período; a avaliação da produção, também estratificada por categoria, por setor da produção ou por período. Exemplos extremos: a objetividade de um míssil alcançar um alvo ou de uma nave espacial alcançar seu objetivo (ambos em porcentagem).

Já o resultado de uma avaliação qualitativa é baseado na interpretação do avaliador sobre o "objeto" avaliado. Segundo Neves (1996), os métodos qualitativos se assemelham a procedimentos de interpretação dos fenômenos que empregamos no nosso dia-a-dia, trata-se de dados simbólicos, situados em determinado contexto, que revelam parte da realidade ao mesmo tempo em que escondem outra parte. $\mathrm{O}$ vínculo entre signo e significado, conhecimento e fenômeno, sempre depende do arcabouço de interpretação empregado pelo investigador, que lhe serve de visão de mundo e de referencial. Assim, avaliadores distintos, ao analisarem ou avaliarem qualitativamente um determinado "objeto", podem extrair resultados distintos, podendo ter de pouca a muita diferença.

Exemplos de avaliações qualitativas: avaliação da motivação dos funcionários, avaliação da qualificação dos funcionários, avaliação do clima organizacional, avaliação da qualidade dos serviços prestados, avaliação da credibilidade de clientes e fornecedores, etc. Em todas essas avaliações, há a interpretação do avaliador dentro de um determinado contexto. Ao final da avaliação, o avaliador atribui um determinado valor que representa a interpretação de sua observação do "objeto" avaliado.

Para Pope e Mays (1995), os métodos quantitativos e qualitativos não se excluem. Embora difiram quanto à forma e à ênfase, os métodos qualitativos trazem como contribuição à investigação uma mistura de procedimentos de cunho racional e intuitivo capazes de contribuir para uma melhor compreensão dos fenômenos ou objetos investigados.

\section{A paraconsistência em avaliações, análises e processos decisóríos}

Drucker (1978) afirma que as decisões só serão boas se forem baseadas no choque de idéias conflitantes, no diálogo entre pontos de vista diferentes, na escolha entre julgamentos diversos. A divergência é necessária, acima de tudo, para estimular a imaginação e para criar soluções inovadoras. A divergência protege o decisor contra ser levado pelo mais óbvio, porém falso ou incompleto. A divergência transforma o plausível em correto e o correto em uma boa decisão. Drucker afirma ainda que todos os presidentes eficazes da história americana tiveram seu método próprio de provocar a divergência que precisavam para tomar uma decisão eficaz. Assim foi com George Washington, Abraham Lincoln, Theodore Roosevelt, Franklin Roosevelt e Harry Truman.

A técnica proposta averigua a divergência de opiniões durante uma avaliação qualitativa. Porém, como utiliza a Lógica Paraconsistente Anotada de Dois Valores, a palavra "divergência" será substituída por "paraconsistência" que é um conceito mais amplo. Na técnica proposta, a paraconsistência estará sempre averiguando se existe algum nível de inconsistência, divergência ou contradição nas diferentes opiniões durante uma avaliação qualitativa, por isso recebe o nome de avaliação qualitativa paraconsistente. A partir do tópico oito ficará mais clara a diferença entre inconsistência, divergência e contradição.

Com relação à contradição, é necessário desde já fazer alguns esclarecimentos. Embora a maioria das ciências evite a contradição, ela está presente em muitos ramos de atividades e do conhecimento. Por exemplo, algumas pessoas são amadas por uns e odiadas por outros, ou seja, essas pessoas são amadas e odiadas ao mesmo tempo, demonstrando um flagrante de contradição. Algo semelhante acontece com determinados locais, são adorados por uns e detestados por outros. Vários outros exemplos podem ser citados: livros; programas de televisão; filmes; músicas; clima e temperatura; veículos; costumes e tradições; política e políticos; esportes; profissionais da saúde, da educação, do jornalismo, da comunicação e do entretenimento; etc. Em todos esses casos, a contradição é uma realidade e tem-se que conviver naturalmente com ela. O problema maior é tomar uma decisão quando existe uma contradição. Este artigo dará a sua contribuição para solucionar esse problema.

A paraconsistência em avaliações, análises e em processos decisórios não é incomum, porém, é pouco abordada e, muitas vezes, desconsiderada. Ou seja, as divergências, contradições e inconsistências estão freqüentemente presentes nessas atividades. Podem-se citar alguns exemplos de paraconsistência nos mais variados ramos de atividade. Na área da Medicina, quando se reúne uma junta médica para decidir qual tratamento dar a um paciente que apresenta um quadro mais delicado ou complicado, não é difícil ocorrerem opiniões médicas divergentes ou totalmente opostas, após a avaliação da situação atual do paciente, e cada grupo propor um tratamento diferente. Neste caso, apesar de existirem paraconsistências entre as opiniões formadas, normalmente a solução é se chegar a um consenso para a decisão final. Se as paraconsistências fossem mais bem trabalhadas, talvez levassem a uma melhor decisão.

$\mathrm{Na}$ área do Direito, a paraconsistência ocorre durante um julgamento quando os advogados da defesa e da acu- 
sação levantam as mais variadas provas, o primeiro tentando defender o réu e o segundo tentando incriminá-lo. Para os componentes do júri popular que têm de tomar a decisão de culpar ou inocentar o réu, as paraconsistências que vão surgindo durante o julgamento tornam cada vez mais difícil suas respectivas decisões, porém o resultado final é muito melhor com do que sem as paraconsistências.

Na política, em época de campanha eleitoral, normalmente, os candidatos criticam a linha de atuação e de conduta dos adversários, proporcionando inúmeras paraconsistências que confundem e dificultam a decisão dos eleitores na escolha do melhor candidato para quem vão confiar seus respectivos votos. Mesmo assim, é preferível a presença das paraconsistências do que a sua ausência.

Nas empresas, as paraconsistências também estão freqüentemente presentes. Por exemplo, para elaborar um novo produto ou uma nova tecnologia, há a necessidade de realizar um estudo de viabilidade para averiguar se o mercado comporta a inovação. Durante este estudo pode ocorrer de se obter dados paraconsistentes, alguns apontando para o sucesso da inovação, demonstrando existir, por exemplo, uma boa receptividade por parte de alguns potenciais clientes. Em contrapartida, como existe uma aversão por parte de outros potenciais clientes a toda e qualquer inovação (o que significaria mudanças e novos gastos), esses dados podem apontar para a inviabilidade da inovação. Essa paraconsistência dificulta a decisão de se levar adiante o projeto da inovação, porém é muito importante que ela esteja presente e, a partir dela, que se analise com mais detalhe a viabilidade da inovação.

Ainda no segmento acima, antes de se efetuar uma compra é necessário se obter o máximo de informações sobre os fornecedores e seus produtos. Podem-se obter dados paraconsistentes de um mesmo fornecedor a partir de fontes de informação distintas, umas fornecendo informações positivas e outras negativas, dificultando a decisão da escolha do fornecedor. Mais uma vez as paraconsistências mostraram sua importância.

Com relação à contratação de novos funcionários por parte do pessoal de Recursos Humanos, acontece algo muito semelhante ao relatado no parágrafo anterior, ou seja, informações paraconsistentes sobre determinados candidatos podem dificultar as análises e o processo decisório do preenchimento dos cargos vagos.

A área de Gestão Ambiental também está sujeita a paraconsistências. Durante a elaboração e implementação de um Sistema de Gestão Ambiental é muito provável que existam opiniões paraconsistentes sobre alguns pontos do projeto, levando em consideração que para elaborá-lo e implementá-lo serão necessários diversos tipos de profissionais e serão envolvidas pessoas das mais variadas correntes de pensamento, comportamento e ação.
As paraconsistências serão proveitosas ao contribuírem para a elaboração e implantação de um melhor Sistema de Gestão Ambiental.

\section{A origem das Lógicas Paraconsistentes}

Para solucionar teoricamente problemas de inconsistências ou contradições em sistemas formais complexos, pesquisadores da Lógica desenvolveram ramificações desta ciência atuando sobre o assunto. O lógico polonês S. Jaskowoski (1904-1965) e o lógico brasileiro Newton da Costa desenvolveram simultaneamente e independentemente os estudos que levaram à criação da Lógica Paraconsistente. Durante mais de uma década, ambos desenvolveram separadamente este novo tipo de lógica e, posteriormente, os trabalhos foram unificados dando mais consistência à nova lógica. Esta lógica foi desenvolvida para solucionar problemas que não podiam ser resolvidos por outras lógicas, inicialmente os sistemas inconsistentes e, posteriormente, o problema da contradição. As lógicas clássicas e a maioria das não clássicas não solucionam problemas que contenham contradição.

Mesmo depois de consolidada, a Lógica Paraconsistente foi sendo aperfeiçoada e surgiram suas ramificações. Entre estas ramificações está a Lógica Paraconsistente Anotada, desenvolvida por Newton da Costa, Jair Minoro Abe e V. S. Subrahmaniam (Costa et al., 1991) e delineada em Abe (1992). Esta lógica permite trabalhar com mais de um valor para a mesma variável, facilitando os cálculos e análises em problemas que contenham paraconsistências.

A Lógica Paraconsistente Anotada de Dois Valores LPA2V (Costa et al., 1999) é uma ramificação da Lógica Paraconsistente Anotada. Em sua técnica de anotação são adotados para cada variável os valores do grau de certeza e de incerteza: $p\left(\mu_{1}, \mu_{2}\right)$. Mais detalhes dessa lógica são descritos no tópico oito.

\section{A norma ISO 14001 e a avaliação de um Sistema de Gestão Ambiental}

Como conseqüência da Conferência Internacional sobre Meio Ambiente (Rio-92), a Confederação das Nações Unidas para o Meio Ambiente e Desenvolvimento Sustentável criou um comitê técnico que elaborou as normas da série ISO 14000. Essa série de normas possui padrões internacionalmente reconhecidos para estruturar todo o Sistema de Gestão Ambiental - SGA (ou EMS Environment Management System) de uma organização e o gerenciamento do desempenho ambiental. Fazem parte dessa série as seguintes normas:

- 14001 - especificações para o SGA;

- 14004 - diretrizes gerais; 
- 19011 - auditoria sobre SGQ e SGA;

- 14020, 14021, 14024 e 14025 - rotulagem;

- 14031 e 14032 - avaliação de desempenho ambiental;

- 14040 a 14043 e 14047 a 14049 - análise de ciclo de vida;

- 14050 - termos e definições;

- 14062 - projetos ambientais; e

- 14063 - comunicação ambiental.

Segundo Oliveira (2004), a série de normas ISO 14000 buscou o alinhamento com a série de normas sobre Qualidade, a série ISO 9000, deixando clara a necessidade de integração entre os conceitos de qualidade e de meio ambiente. A base para a elaboração de um Sistema de Gestão Ambiental é a mesma utilizada para a elaboração do Sistema de Gestão da Qualidade. Essa equivalência entre os modelos facilita o trabalho das organizações que tenham como objetivo melhorar a qualidade ambiental de seus processos.

A norma ISO 14001 é o documento base da série de normas ISO 14000 para um Sistema de Gestão Ambiental, somente ela é certificada e as demais são normas auxiliares. Ela foi elaborada após o sucesso dos padrões de qualidade da série ISO 9000. A certificação pela ISO 14001: 2004 é uma validação reconhecida das conformidades de uma organização de seu Sistema de Gestão Ambiental (SGA) em relação aos padrões dessa norma, por uma empresa certificadora independente. A auditoria de certificação tem como objetivo verificar se o SGA da organização está conforme os padrões da ISO 14001: 2004.

Entre os benefícios para a empresa certificada estão: satisfação de necessidades contratuais (principalmente para exportação); expansão de mercados e da base de clientes; maior competitividade; melhoria da imagem principalmente para clientes, fiscalização, funcionários, investidores e para as comunidades envolvidas com a empresa.

A versão brasileira da norma ISO 14001: 2004 é a NBR ISO 14001: 2004. Tanto a versão internacional quanto a brasileira tiveram sua primeira versão em 1996, ambas sofreram uma atualização em 2001 e tiveram uma nova versão em 2004. Segundo a própria norma, sua nova versão focou a melhoria do entendimento de alguns requisitos e, também, o aumento da compatibilidade com a norma ISO 9001: 2000.

Segundo a BVQI (2004), o Brasil ocupa a $15^{\mathrm{a}}$ posição no ranking mundial com 1.500 empresas certificadas pela norma, no mundo são pouco mais de 61.000 .

A certificação pela ISO 14001: 2004 é alcançada por um processo de cinco etapas:

1) solicitação do registro;

2) revisão da documentação do SGA;
3) revisão preliminar no local;

4) auditoria de certificação; e

5) determinação da certificação.

Sem a pretensão de analisar ou discutir os detalhes da norma, serão apresentados seus principais itens, os quais deverão ser elaborados e implementados durante a implantação do sistema. A apresentação daqueles itens visa facilitar a compreensão da aplicação da avaliação qualitativa paraconsistente proposta.

Em entrevista com Marcos Alberto de Oliveira, auditor de Sistemas da Qualidade ISO 9000 Lead Assessor pela BVQI, ao lhe ser apresentada uma versão prévia deste artigo, afirmou que as avaliações necessárias para a implantação de sistemas de qualidade, independente de qual seja, assim como a própria avaliação para certificação, já possuem uma estrutura bem estabelecida e consagrada. Porém, segundo o auditor, de fato são feitas algumas análises qualitativas complementares para auxiliar aquelas avaliações. Com relação à técnica proposta, o auditor afirmou que de fato ela pode e deve contribuir para a avaliação global e dos itens isolados, mas só a prática poderia dizer o nível de sua aplicabilidade.

Pelo tipo de avaliação do sistema praticado atualmente, na maioria dos itens avaliados, verifica-se se estes foram cumpridos e se estão em conformidade com a norma e com o restante do sistema. Uma avaliação predominantemente quantitativa.

Maimon (1999) foi a única publicação encontrada que abordou a avaliação desse sistema de forma qualitativa. Propôs-se o preenchimento da Tabela 1 para avaliar o grau de esforço que uma empresa deverá empreender para implantar um Sistema de Gestão Ambiental, a partir do ponto em que se encontra a empresa atualmente com relação à sua Gestão Ambiental. Na publicação, há maiores detalhes dos aspectos que devem ser observados para avaliar cada item. Para cada item constante na tabela é necessário se atribuir uma nota que varia de 1 a 5. Portanto, trata-se de uma avaliação totalmente qualitativa, porém incompleta do ponto de vista da avaliação qualitativa paraconsistente, como ficará demonstrado no artigo.

A implantação do Sistema de Gestão Ambiental é totalmente independente de uma futura certificação. As etapas necessárias para sua implantação são maiores que os da certificação, porém, em todas essas etapas têm-se como meta aquela certificação. Essas etapas são:

- o comprometimento e colaboração de todos na empresa, desde a alta direção até os empregados mais simples;

- fazer uma avaliação das práticas e procedimentos ambientais já existentes;

- planejar a migração para o novo sistema; e

- implementá-lo. 
Tabela 1. Grau de esforço que a empresa deverá empreender para implantar um SGA (Maimom, 1999, p. 18).

\begin{tabular}{l}
\hline \multicolumn{1}{c}{ Item avaliado } \\
\hline 1. Política Ambiental \\
2. Aspectos Ambientais \\
3. Requisitos Legais \\
4. Objetivos e Metas \\
5. Gestão da Qualidade do Ar \\
6. Gestão da Qualidade da Água \\
7. Gestão do Consumo de Água \\
8. Gestão de Resíduos \\
9. Gestão de Produtos Perigosos \\
10. Gestão de Consumo de Energia \\
11. Alocação de Recursos \\
12. Atribuições e Responsabilidades \\
13. Conscientização e Treinamento \\
14. Comunicação Interna \\
15. Comunicação Externa \\
16. Documentação \\
17. Controle Operacional \\
18. Ações de Emergência \\
19. Medições \\
20. Auditorias Ambientais \\
21. Melhoria Contínua
\end{tabular}

\section{A avaliação qualitativa tradicional}

A Tabela 2 apresenta uma simulação da avaliação qualitativa tradicional da implantação de um Sistema de Gestão Ambiental com base na norma NBR ISO 14001: 2004. Como a avaliação é qualitativa, está sendo atribuída uma nota que varia de 0 a 10 para cada item avaliado. Os itens de 1 a 3, apesar de constarem da norma e, em conseqüência, da Tabela 2, não apresentam notas, devido ao fato de a norma prever que eles são considerados itens apenas de apresentação. Portanto, apenas o item 4 é avaliado e a nota final de toda a avaliação é a nota deste item, que é a média dos itens de 4.1 a 4.6. Se há subitens (por exemplo 4.4.1, 4.4.2, ...) o item que os agrupa (4.4) apresenta a média de suas notas.

A simulação da avaliação qualitativa tradicional resultou em uma média final de 8,5. Isto significa afirmar que o grau de confiança ou de certeza da qualidade do sistema avaliado é de $85 \%$. Automaticamente o grau de falta de confiança ou de incerteza de sua qualidade é de $15 \%$. Desta forma, este sistema poderia ser classificado como ótimo.

Porém, se aquele grau de falta de confiança ou de incerteza fosse calculado não automaticamente, mas sim de forma separada e independente do grau de confiança ou de certeza, muito provavelmente não seriam obtidos
Tabela 2. Simulação da avaliação qualitativa tradicional da implantação de um Sistema de Gestão Ambiental com base na norma NBR ISO 14001: 2004.

\begin{tabular}{lc}
\hline \multicolumn{1}{c}{ Item avaliado } & Nota \\
\hline 1. Objetivos & - \\
2. Referências Normativas & - \\
3. Definições & - \\
4. Requisitos do Sistema de Gestão Ambiental & $\mathbf{8 , 5 0}$ \\
4.1 Requisitos Gerais & $\mathbf{8 , 5 0}$ \\
4.2 Política Ambiental & $\mathbf{9 , 0 0}$ \\
4.3 Planejamento & $\mathbf{8 , 6 7}$ \\
4.3.1 Aspectos Ambientais & 8,00 \\
4.3.2 Requisitos Legais e Outros Requisitos & 9,00 \\
4.3.3 Objetivos, Metas e Programa de Gestão & 9,00 \\
Ambiental & \\
4.4 Implementação e Operação do Sistema de & $\mathbf{8 , 4 3}$ \\
Gestão Ambiental & \\
4.4.1 Estrutura e Responsabilidade & 8,00 \\
4.4.2 Treinamento, Conscientização & 8,50 \\
e Competência & \\
4.4.3 Comunicação & 8,50 \\
4.4.4 Documentação do Sistema de Gestão & 8,00 \\
Ambiental & \\
4.4.5 Controle de documentos & 9,00 \\
4.4.6 Controle Operacional & 9,00 \\
4.4.7 Preparação e Atendimento a Emergências & 8,00 \\
4.5 Verificação e Ação Corretiva & $\mathbf{8 , 4 0}$ \\
4.5.1 Monitoramento e Medição & 8,00 \\
4.5.2 Avaliação de Conformidade & 9,00 \\
4.5.3 Não-conformidade e ações corretivas e & 8,50 \\
preventivas & $\mathbf{8 , 0 0}$ \\
4.5.4 Registros & \\
4.5.5 Auditoria do Sistema de Gestão Ambiental & 8,00 \\
4.6 Análise Crítica pela Administração & \\
\hline & \\
&
\end{tabular}

os mesmos $15 \%$. Se, em uma segunda avaliação qualitativa, em que se medissem as faltas ou as falhas de qualidade de cada item do sistema, de uma forma totalmente separada e independente da primeira avaliação, é muito provável que o valor do grau de incerteza fosse diferente do obtido na primeira avaliação. Se o valor do grau de incerteza obtido na segunda avaliação fosse inferior ao da primeira avaliação, reforçaria a tese de que se trata de um ótimo sistema. Se o valor obtido na segunda avaliação fosse igual ou muito próximo aos $15 \%$, também reforçaria a qualidade daquele sistema e demonstraria que a primeira avaliação qualitativa foi muito bem feita. Porém, se o valor do grau de incerteza obtido na segunda avaliação for muito superior aos $15 \%$, já temos um cenário paraconsistente. $\mathrm{O}$ fato do valor do grau de incerteza obtido na segunda avaliação ser superior aos $15 \%$ obtidos na primeira avaliação, não invalida a primeira avaliação, 
apenas reforça a existência de uma paraconsistência e a necessidade de uma investigação mais profunda para tentar descobrir sua causa.

\section{A avaliação qualitativa paraconsistente}

Em Bispo e Cazarini (2004) afirma-se que a idéia da dupla avaliação assemelha-se a um julgamento judicial. Há a necessidade de que o grau de certeza seja avaliado por uma determinada equipe que levantará o nível da qualidade do sistema. Essa equipe fará o papel do advogado de defesa do sistema, defendendo a sua qualidade. Uma outra equipe fará a avaliação que determinará o nível da falta de qualidade do sistema e/ou suas falhas, ou seja, o seu grau de incerteza. Essa outra equipe fará o papel do advogado de acusação do sistema, defendendo a sua falta de qualidade. Se fosse utilizada a mesma equipe para fazer as duas avaliações, seus resultados não poderiam ser considerados honestos, pois poderia haver a tendência de os resultados da avaliação do grau de incerteza terem os valores complementares a $100 \%$ em relação aos valores obtidos pelo grau de certeza, descaracterizando o propósito da dupla avaliação. O confronto entre as duas avaliações proporcionará o real grau de qualidade do sistema, diferente do resultado apresentado quando se trabalha apenas com o levantamento do grau de certeza ou de confiança na qualidade do sistema.

Além de proporcionar uma avaliação mais real da qualidade do sistema, a dupla avaliação permite averiguar se existem paraconsistências nos itens avaliados e em quais deles. Identificadas as paraconsistências, primeiro é necessário averiguar eventuais falhas na dupla avaliação. Tendo em vista que ambas são qualitativas, a interpretação pessoal de cada avaliador, dentro de seu próprio contexto, está embutida automaticamente em cada resultado fornecido. Esse contexto é formado principalmente pelo conhecimento específico sobre o item avaliado; o conhecimento sobre as interconexões com outros itens e assuntos coligados; e pelos fatores de influência de caráter técnico, político, profissional e pessoal. Tudo isso combinado reflete muito na interpretação do resultado da avaliação e pode levar o avaliador a cometer pequenas falhas ou falhas mais significantes. Revistas as avaliações, se não houver falhas, é necessário investigar se há algo errado com cada item que apresentou paraconsistência ou se são itens paraconsistentes (contraditórios) por natureza.

Uma nova simulação da avaliação dos mesmos itens, desta vez avaliando suas respectivas falta de qualidade ou falhas, está sendo mostrada na Tabela 3. Nesta tabela, os itens de 1 a 3 estão (que não são avaliados) sendo ocultados por falta de espaço. Nessa nova simulação, a avaliação final atribuída foi de 4,08, ou seja, o grau de incerteza foi de $40,8 \%$. Sabe-se que em uma simulação podem-se
Tabela 3. Simulação dos graus de incerteza em uma avaliação qualitativa realizada de forma separada e independente da avaliação qualitativa tradicional.

\begin{tabular}{lc}
\hline \multicolumn{1}{c}{ Item avaliado } & Nota \\
\hline 4. Falhas nos Requisitos do & $\mathbf{4 , 0 8}$ \\
Sistema de Gestão Ambiental & \\
4.1 Falhas nos Requisitos Gerais & $\mathbf{3 , 3 0}$ \\
4.2 Falhas na Política Ambiental & $\mathbf{6 , 2 0}$ \\
4.3 Falhas no Planejamento & $\mathbf{3 , 5 0}$ \\
4.3.1 Falhas nos Aspectos Ambientais & 3,60 \\
4.3.2 Falhas nos Requisitos Legais e Outros & 3,50 \\
Requisitos & \\
4.3.3 Falhas nos Objetivos, Metas e & 3,40 \\
Programa de Gestão Ambiental & \\
4.4 Falhas na Implementação e Operação do & $\mathbf{3 , 7 7}$ \\
Sistema de Gestão Ambiental & \\
4.4.1 Falhas na Estrutura e Responsabilidade & 3,50 \\
4.4.2 Falhas no Treinamento, & 4,00 \\
Conscientização e Competência & \\
4.4.3 Comunicação & 3,50 \\
4.4.4 Falhas na Documentação & 3,80 \\
do Sistema de Gestão Ambiental & \\
4.4.5 Falhas no Controle de documentos & 3,60 \\
4.4.6 Falhas no Controle Operacional & 4,00 \\
4.4.7 Falhas na Preparação e Atendimento a & 4,00 \\
Emergências & \\
4.5 Falhas na Verificação e Ação Corretiva & 3,700 \\
4.5.1 Falhas no Monitoramento e Medição & 3,70 \\
4.5.2 Falhas na Avaliação de Conformidade & \\
4.5.3 Falhas na Não-conformidade e & \\
ações corretivas e preventivas & \\
4.5.4 Falhas nos Registros & \\
4.5.5 Falhas na Auditoria do Sistema de Gestão & 4,00 \\
\hline
\end{tabular}

manipular os dados de forma a produzir os resultados almejados. Porém, em uma situação real, acredita-se que as paraconsistências também ocorrerão com frequiência, reforçando a necessidade dessa dupla avaliação.

A Tabela 4 apresenta os resultados obtidos de cada item nas duas avaliações. Nela é possível confrontá-los, averiguar se existem e localizar as paraconsistências. Para que a tabela não ficasse muito extensa, está sendo exibida apenas a numeração de cada item.

Pode-se notar que todos o itens, ao serem comparadas as duas avaliações, apresentaram algum nível de disparidade. Na prática, seguindo as recomendações da técnica, dificilmente dois avaliadores ou dois grupos de avaliadores, um avaliando o grau de certeza e outro o grau de incerteza de forma completamente independente, chegarão a resultados iguais aos da avaliação tradicional. Portanto, todas ou quase todas as avaliações apresentarão uma pa- 
Tabela 4. Dupla avaliação qualitativa da implantação de um Sistema de Gestão Ambiental com base na norma NBR ISO 14001: 2004.

\begin{tabular}{ccccc}
\hline Itens avaliados & \multicolumn{2}{c}{ Avaliação tradicional } & \multicolumn{2}{c}{$\begin{array}{c}\text { Avaliação paraconsistente } \\
\text { Grau de certeza }\end{array}$} \\
\hline & Grau de certeza & Grau de incerteza & - & - \\
\hline $\mathbf{1 .}$ & - & - & - & - \\
$\mathbf{2 .}$ & - & - & - & - \\
$\mathbf{3 .}$ & - & - & $\mathbf{8 , 5 0}$ & $\mathbf{4 , 0 8}$ \\
$\mathbf{4 .}$ & $\mathbf{8 , 5 0}$ & $\mathbf{1 , 5 0}$ & $\mathbf{8 , 5 0}$ & $\mathbf{3 , 3 0}$ \\
4.1 & $\mathbf{8 , 5 0}$ & $\mathbf{1 , 5 0}$ & $\mathbf{9 , 0 0}$ & $\mathbf{6 , 2 0}$ \\
4.2 & $\mathbf{9 , 0 0}$ & $\mathbf{1 , 0 0}$ & $\mathbf{8 , 6 7}$ & 3,60 \\
4.3 & $\mathbf{8 , 6 7}$ & $\mathbf{1 , 3 3}$ & 8,00 & 3,50 \\
4.3 .1 & 8,00 & 2,00 & 9,00 & 3,40 \\
4.3 .2 & 9,00 & 1,00 & 9,00 & $\mathbf{3 , 7 7}$ \\
4.3 .3 & 9,00 & 1,00 & $\mathbf{8 , 4 3}$ & 3,50 \\
4.4 & $\mathbf{8 , 4 3}$ & $\mathbf{1 , 5 7}$ & 8,00 & 4,00 \\
4.4 .1 & 8,00 & 2,00 & 8,50 & 3,50 \\
4.4 .2 & 8,50 & 1,50 & 8,50 & 3,80 \\
4.4 .3 & 8,50 & 1,50 & 8,00 & 3,60 \\
4.4 .4 & 8,00 & 2,00 & 9,00 & 4,00 \\
4.4 .5 & 9,00 & 1,00 & 9,00 & 4,00 \\
4.4 .6 & 9,00 & 1,00 & 8,00 & $\mathbf{3 , 7 0}$ \\
4.4 .7 & 8,00 & 2,00 & $\mathbf{8 , 4 0}$ & 3,60 \\
4.5 & $\mathbf{8 , 4 0}$ & $\mathbf{1 , 6 0}$ & 8,00 & 3,70 \\
4.5 .1 & 8,00 & 2,00 & 9,00 & 3,70 \\
4.5 .2 & 9,00 & 1,00 & 8,50 & 3,50 \\
4.5 .3 & 8,50 & 1,50 & 8,50 & 4,00 \\
4.5 .4 & 8,50 & 1,50 & 8,00 & $\mathbf{4 , 0 0}$ \\
4.5 .5 & 8,00 & 2,00 & $\mathbf{8 , 0 0}$ &
\end{tabular}

raconsistência em algum grau. Mas será que é necessário se investigar todas as paraconsistências? É obvio que não, existem valores que são toleráveis. Quais são esses valores? Depende da precisão do que está sendo avaliado. Para auxiliar essas questões e a definição do real nível de qualidade do sistema avaliado, a Lógica Paraconsistente Anotada de Dois Valores possui ferramentas apropriadas.

\section{As ferramentas para auxiliar a avaliação qualitativa paraconsistente}

A técnica da avaliação qualitativa paraconsistente foi criada a partir da Lógica Paraconsistente Anotada de Dois Valores - LPA2V (Costa et al., 1999). Essa lógica é uma ramificação da Lógica Paraconsistente Anotada e, em sua técnica de anotação, são adotados, para cada variável, os valores do grau de certeza e de incerteza: $p\left(\mu_{1}, \mu_{2}\right)$. A variável $p$ representa aquilo que está sendo avaliado, a anotação $\mu_{1}$ representa seu grau de crença ou de certeza e $\mu_{2}$, o seu grau de descrença ou de incerteza.

\subsection{As ferramentas da LPA2V}

Segundo Costa et al. (1999), após a obtenção dos valores dos graus de certeza e de incerteza, a Lógica Paraconsistente Anotada de Dois Valores - LPA2V possui duas ferramentas distintas para classificar aqueles valores em doze resultados distintos:

- v: verdadeiro;

- f: falso;

- $\perp$ : indeterminado;

- $\mathrm{T}$ : inconsistente;

- $\mathrm{T} \rightarrow \mathrm{f}$ : inconsistente tendendo ao falso;

- $\mathrm{T} \rightarrow \mathrm{v}$ : inconsistente tendendo ao verdadeiro;

- $\perp \rightarrow \mathrm{f}$ : indeterminado tendendo ao falso;

- $\perp \rightarrow \mathrm{v}$ : indeterminado tendendo ao verdadeiro;

- Qf $\rightarrow \mathrm{T}$ : quase falso tendendo ao inconsistente;

- Qf $\rightarrow \perp$ : quase falso tendendo ao indeterminado;

- $\mathrm{Qv} \rightarrow \mathrm{T}$ : quase verdadeiro tendendo ao inconsistente; $\mathrm{e}$

- $\mathrm{Qv} \rightarrow \perp$ : quase verdadeiro tendendo ao indeterminado. 
Desses doze resultados possíveis, quatro são valores extremos e os únicos utilizados nas avaliações qualitativas tradicionais, são eles: verdadeiro, falso, indeterminado e inconsistente. Porém, nestas avaliações, na prática, quando há uma paraconsistência, ou seja, uma divergência, uma contradição ou uma inconsistência, essas são desconsideradas ou menosprezadas se o sistema avaliado puder prosseguir sem elas, mesmo que apresentem resultados um pouco mais distantes da realidade. Os oito valores intermediários são exclusividade da LPA2V e constituem as divergências. Com todos esses valores, essa lógica permite trabalhar naturalmente com divergências, inconsistências e contradições e permite obter resultados mais precisos e mais próximos da realidade.

Uma das ferramentas para se obter os resultados acima é o algoritmo para-analisador e a outra é o quadrado unitário do plano cartesiano, ambas publicadas em Costa et al. (1999).

\subsection{O algoritmo para-analisador da LPA2V}

Este algoritmo permite uma automação computacional da averiguação dos resultados da variável que está sendo analisada. As variáveis que serão utilizadas internamente dentro do algoritmo são:

- $\mathbf{G}_{\mathrm{it}}$ : grau de inconsistência, em que $\mathbf{G}_{\mathrm{it}}=\mu_{1}+\mu_{2}-\mathbf{1}$, se e somente se, $\left(\mu_{1}+\mu_{2}\right) \geq \mathbf{1}$;

- $\mathbf{G}_{\text {idd }}$ : grau de indeterminação, em que $\mathbf{G}_{\mathbf{i d}}=\mu_{1}+\mu_{2}$ $-\mathbf{1}$, se e somente se, $\left(\mu_{1}+\mu_{2}\right) \leq \mathbf{1}$;

- $\mathbf{G}_{v}$ : grau de verdade, em que $\mathbf{G}_{v}=\mu_{1}-\mu_{2}$, se e somente se, $\mu_{1} \geq \mu_{2}$;

- $\mathbf{G}_{f}$ : grau de falsidade, em que $\mathbf{G}_{f}=\mu_{1}-\mu_{2}$, se e somente se, $\mu_{1} \leq \mu_{2}$;

- $\mathbf{G}_{\mathrm{ct}}$ : grau de contradição, em que $\mathbf{G}_{\mathrm{ct}}=\mu_{1}+\mu_{2}-\mathbf{1}$, se e somente se, $\mathbf{0} \leq \mu_{1} \leq \mathbf{1}$ e $\mathbf{0} \leq \mu_{2} \leq \mathbf{1}$;

- $\mathbf{G}_{\mathbf{c}}$ : grau de certeza, em que $\mathbf{G}_{\mathbf{c}}=\mu_{1}-\mu_{2}$, se e somente se, $\mathbf{0} \leq \mu_{1} \leq \mathbf{1}$ e $\mathbf{0} \leq \mu_{2} \leq \mathbf{1}$.

$\mathrm{O}$ algoritmo para-analisador, adaptado de Costa et al. (1999, p. 80-81), é:

*/ Variáveis de entrada (valores fornecidos pelo usuário)*/

$\mu_{1} \quad * /$ grau de certeza $* /$

$\mu_{2} \quad * /$ grau de incerteza $* /$

$\mathbf{V}_{\text {scc }}$ (valor padrão $\left.=0,5\right) \quad * /$ valor superior de controle de certeza $* /$

$\mathbf{V}_{\text {scet }}$ (valor padrão $=0,5$ ) controle de contradição */

$\mathbf{V}_{\text {icc }}$ (valor padrão $=-0,5$ ) controle de certeza */

$\mathbf{V}_{\text {icct }}$ (valor padrão $\left.=-0,5\right)$ controle de contradição */

*/ Variável de saída (resultado fornecido pelo algoritmo)*/
S

*/ Cálculo do valor da variável de saída */

Se $\mathbf{0} \leq \mu_{1} \leq \mathbf{1}$ e $\mathbf{0} \leq \mu_{2} \leq \mathbf{1}$ então

$\mathbf{G}_{\mathrm{ct}}=\mu_{1}+\mu_{2}-\mathbf{1} \mathbf{e} \mathbf{G}_{\mathbf{c}}=\mu_{1}-\mu_{2}$

Se $G_{c} \geq V_{\text {scc }}$ então $S=v$

Se $G_{c} \leq V_{\text {icc }}$ então $S=f$

Se $G_{\text {ct }} \geq V_{\text {sct }}$ então $S=T$

$\operatorname{Se~} G_{\text {ct }} \geq V_{\text {icct }}^{\text {scct }}$ então $S=\perp$

Se $\mathbf{0} \leq \mathbf{G}_{\mathrm{c}}<\mathbf{V}_{\text {scc }}$ e $\mathbf{0} \leq \mathbf{G}_{\text {ct }}<\mathbf{V}_{\text {scct }}$ então

$\operatorname{Se} \mathbf{G}_{\mathbf{c}} \geq \mathbf{G}_{\mathrm{ct}}$ então $\mathbf{S}=\mathbf{Q}_{v} \rightarrow \mathbf{T}$

$\operatorname{Se} \mathbf{G}_{\mathbf{c}}<\mathbf{G}_{\mathrm{ct}}$ então $\mathbf{S}=\mathbf{T} \rightarrow \boldsymbol{v}$

Se $\mathbf{0} \leq \mathbf{G}_{\mathrm{c}}<\mathbf{V}_{\text {scc }}$ e $\mathbf{V}_{\text {icct }}<\mathbf{G}_{\text {ct }} \leq \mathbf{0}$ então

$\operatorname{Se} \mathbf{G}_{\mathbf{c}} \geq\left|\mathbf{G}_{\mathrm{ct}}\right|$ então $\mathbf{S}=\mathbf{Q}_{\mathrm{v}} \rightarrow \perp$

$\operatorname{Se} \mathbf{G}_{\mathrm{c}}<\left|\mathbf{G}_{\mathrm{ct}}\right|$ então $\mathbf{S}=\perp \rightarrow \boldsymbol{v}$

Se $\mathbf{V}_{\text {icc }}<\mathbf{G}_{\mathrm{c}}<\mathbf{0}$ e $\mathbf{V}_{\text {icct }}<\mathbf{G}_{\text {ct }} \leq \mathbf{0}$ então

Se $\left|\mathbf{G}_{\mathrm{c}}\right| \geq\left|\mathbf{G}_{\mathrm{ct}}\right|$ então $\mathbf{S}=\mathbf{Q}_{f} \rightarrow \perp$

$\operatorname{Se}\left|\mathbf{G}_{\mathbf{c}}\right|<\left|\mathbf{G}_{\mathrm{ct}}\right|$ então $\mathbf{S}=\perp \rightarrow \boldsymbol{f}$

Se $V_{\text {icc }}<\mathbf{G}_{\mathbf{c}} \leq 0$ e $0 \leq \mathbf{G}_{\text {ct }}<\mathbf{V}_{\text {scct }}$ então

$\operatorname{Se}\left|\mathbf{G}_{\mathrm{c}}\right| \geq \mathbf{G}_{\text {ct }}$ então $\mathbf{S}=\mathbf{Q}_{f} \rightarrow \mathbf{T}$

Se $\left|\mathbf{G}_{\mathrm{c}}\right|<\mathbf{G}_{\mathrm{ct}}$ então $\mathbf{S}=\mathbf{T} \rightarrow \boldsymbol{f}$

As variáveis $\mathbf{V}_{\text {scc }}, \mathbf{V}_{\text {scet }}, \mathbf{V}_{\text {icc }}$ e $\mathbf{V}_{\text {icct }}$ permitem ajustes na delimitação dos níveis dos resultados extremos, ou seja, o verdadeiro, o falso, a contradição e a inconsistência, permitindo uma tolerância maior ou menor desses resultados.

\subsection{0 quadrado unitário do plano cartesiano}

A outra ferramenta adotada pela LPA2V é o quadrado unitário do plano cartesiano - QUPC, conforme mostra a Figura 1. Por meio dessa ferramenta pode-se chegar aos mesmos resultados do algoritmo para-analisador. Sua vantagem está na representação gráfica dos resultados, que permite uma percepção mais rápida do cenário a ser analisado.

Como, originalmente, tanto o algoritmo para-analisador como o QUPC trabalham com valores entre 0 e 1 , e as notas atribuídas nas duas simulações variam de 0 a 10 , houve a necessidade dos ajustes. Os valores submetidos ao algoritmo foram divididos por 10, enquanto o QUPC foi ajustado para trabalhar com valores entre 0 e 10 .

\section{Comparação entre os resultados obtidos pelas duas avaliações}

Usando os dados apresentados nas duas avaliações simuladas neste artigo, poder-se-á fazer uma comparação entre seus resultados. A Tabela 4 permite uma comparação entre os dados obtidos pelas duas avaliações simuladas. A Tabela 5 apresenta a classificação desses dados, segundo a LPA2V, calculados pelo algoritmo para-analisador.

Pela simulação da avaliação qualitativa tradicional, o valor do grau de certeza da avaliação final do sistema é 


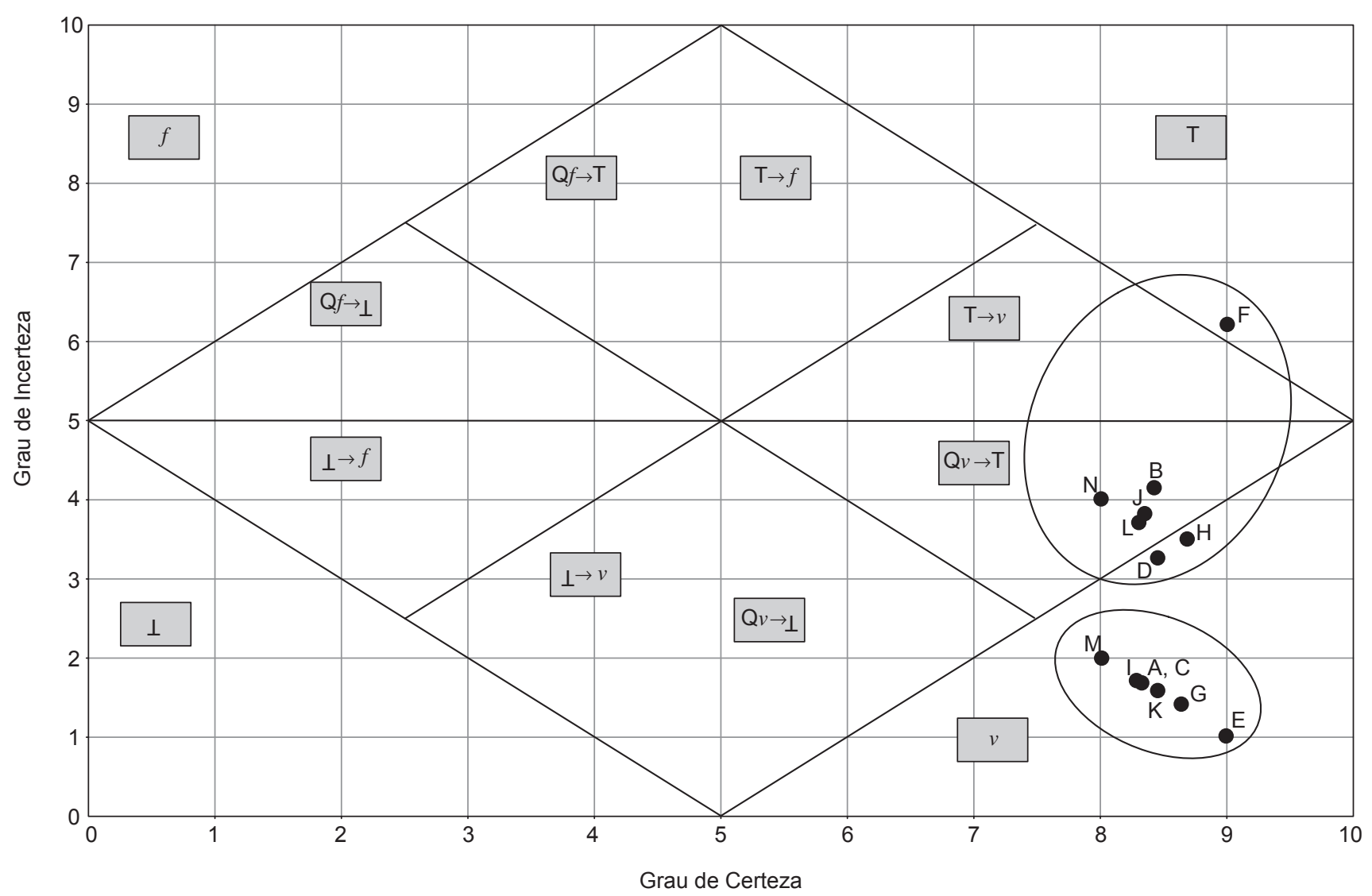

Figura 1. Principais itens das avaliações qualitativas tradicional e paraconsistente.

de $85 \%$, automaticamente atribuindo-lhe o valor do grau de incerteza em $15 \%$. Plotando esse valor no QUPC, obtém-se o ponto $\boldsymbol{A}$ da Figura 1, apresentando o resultado verdadeiro, demonstrando ser uma verdade absoluta a qualidade da implantação do SGA avaliado.

Adotando-se a simulação da avaliação qualitativa paraconsistente, o valor do grau de incerteza passou a ser de 40,8\%. Plotando-o no QUPC, chega-se ao ponto $\boldsymbol{B}$ da Figura 1, apresentando o resultado quase verdadeiro tendendo ao inconsistente. Agora, mostrando um cenário diferente de quando foi utilizada a avaliação qualitativa tradicional, a avaliação qualitativa paraconsistente demonstra que já não é uma verdade absoluta a conclusão de que o sistema avaliado apresenta uma ótima qualidade.

Para se realizar uma avaliação mais detalhada, basta analisar os resultados apresentados nas Tabelas 4 e 5 . Para não sobrecarregar a visualização dos dados no QUPC, plotaram-se apenas os principais valores, mostrando os resultados apresentados na Figura 1. A visualização gráfica daqueles resultados permite averiguá-los e analisálos melhor, demonstrando que o cenário da avaliação tradicional (marcado pela elipse inferior) é bem distinto do cenário da avaliação paraconsistente (marcado pela elip- se superior). Embora não tenham sido plotados os demais itens, porém, de acordo com a Tabela 5 e plotando-os no QUPC, todos os valores da avaliação qualitativa tradicional estão dentro ou muito próximos da elipse inferior, e todos os valores da avaliação qualitativa paraconsistente estão dentro ou muito próximos da elipse superior. Se a avaliação qualitativa paraconsistente realizada fosse real, ela estaria bem mais próxima da realidade e necessitaria de uma intervenção no Sistema de Gestão Ambiental avaliado naqueles itens em que existiram grandes disparidades entre as duas avaliações.

Analisando com detalhes os resultados apresentados, pode-se notar que no item 4.2 houve uma grande disparidade entre as duas avaliações. $\mathrm{Na}$ avaliação qualitativa tradicional, a nota atribuída ao grau de certeza foi 9,0, automaticamente seu grau de incerteza foi de 1,0, plotando no QUPC, o ponto $\boldsymbol{E}$ da Tabela 5 cai na região do verdadeiro. $\mathrm{Na}$ avaliação paraconsistente, o valor do grau de certeza é o mesmo 9,0, já a nota atribuída ao grau de incerteza foi 6,2. Plotando no QUPC, verifica-se que cai na região da contradição o, ponto $\boldsymbol{F}$ da Tabela 5 e do QUPC. Neste caso, faz-se necessária toda a averiguação e investigação já mencionada anteriormente. 
Tabela 5. Resultados da dupla avaliação qualitativa da qualidade de um SGA.

\begin{tabular}{|c|c|c|c|c|}
\hline \multirow[t]{2}{*}{ Itens avaliados } & \multicolumn{2}{|c|}{ Avaliação tradicional } & \multicolumn{2}{|c|}{ Avaliação paraconsistente } \\
\hline & Ponto no QUPC & Resultado da LPA2V & Ponto no QUPC & Resultado da LPA2V \\
\hline 1. & - & - & - & - \\
\hline 2. & - & - & - & - \\
\hline 3. & - & - & - & - \\
\hline 4. & $\mathbf{A}$ & $v$ & B & $\mathrm{Q} v \rightarrow \mathrm{T}$ \\
\hline 4.1 & $\mathbf{C}$ & $v$ & D & $v$ \\
\hline 4.2 & $\mathbf{E}$ & $v$ & $\mathbf{F}$ & $\mathbf{T}$ \\
\hline 4.3 & $\mathbf{G}$ & $v$ & $\mathbf{H}$ & $v$ \\
\hline 4.3.1 & - & $v$ & - & $\mathrm{Q} v \rightarrow \mathrm{T}$ \\
\hline 4.3 .2 & - & $v$ & - & $v$ \\
\hline 4.3.3 & - & $v$ & - & $v$ \\
\hline 4.4 & I & $v$ & $\mathbf{J}$ & $\mathrm{Q} v \rightarrow \mathrm{T}$ \\
\hline 4.4.1 & - & $\mathrm{v}$ & - & $\mathrm{Q} v \rightarrow \mathrm{T}$ \\
\hline 4.4 .2 & - & $v$ & - & $\mathrm{Q} v \rightarrow \mathrm{T}$ \\
\hline 4.4 .3 & - & $v$ & - & $\mathrm{Q} v \rightarrow \mathrm{T}$ \\
\hline 4.4 .4 & - & $v$ & - & $\mathrm{Q} v \rightarrow \mathrm{T}$ \\
\hline 4.4 .5 & - & $v$ & - & $v$ \\
\hline 4.4.6 & - & $v$ & - & $\mathrm{Q} v \rightarrow \mathrm{T}$ \\
\hline 4.4.7 & - & $v$ & - & $\mathrm{Q} v \rightarrow \mathrm{T}$ \\
\hline 4.5 & $\mathbf{K}$ & $v$ & $\mathbf{L}$ & $\mathrm{Q} v \rightarrow \mathrm{T}$ \\
\hline 4.5.1 & - & $v$ & - & $\mathrm{Q} v \rightarrow \mathrm{T}$ \\
\hline 4.5.2 & - & $v$ & - & $v$ \\
\hline 4.5 .3 & - & $v$ & - & $\mathrm{Q} v \rightarrow \mathrm{T}$ \\
\hline 4.5 .4 & - & $v$ & - & $\mathrm{Q} v \rightarrow \mathrm{T}$ \\
\hline 4.5 .5 & - & $v$ & - & $\mathrm{Q} v \rightarrow \mathrm{T}$ \\
\hline 4.6 & M & $v$ & $\mathbf{N}$ & $\mathbf{Q} v \rightarrow \mathrm{T}$ \\
\hline
\end{tabular}

\section{Conclusões}

Numa análise final, poderia ser afirmado que, pela avaliação qualitativa tradicional, o sistema simuladamente avaliado possuía uma ótima qualidade. A avaliação qualitativa paraconsistente demonstrou que essa qualidade não era tão boa assim. Se fosse realizada apenas a avaliação tradicional, alguns problemas não seriam detectados. A contradição ocorrida no item 4.2 - Política Ambiental - jamais seria observada.

Embora o artigo apresente uma simulação controlada das duas avaliações, levando propositalmente aos valores e resultados obtidos, a falha ocorrida no item 4.2 - Política ambiental - pode estar realmente ocorrendo em muitas empresas, nas mais variadas situações. Embora a falha detectada no item tenha sido obtida por meio de uma simulação, o problema da paraconsistência levantado é real e pode afetar qualquer empresa e qualquer sistema.

Como se trata de uma avaliação qualitativa, na prática, a forma como foi avaliado o SGA neste artigo pode necessitar de ajustes, como por exemplo a atribuição de pesos aos itens mais importantes ou a avaliação apenas dos itens que mereçam esse tipo de avaliação, deixando os itens que podem ser melhor avaliados quantitativamente fora da avaliação qualitativa paraconsistente. $\mathrm{O}$ importante é obter resultados o mais próximos possível da realidade e poder investigar as grandes disparidades mostradas na avaliação.

No artigo, demonstrou-se de uma forma bem clara e objetiva que as avaliações qualitativas paraconsistentes podem proporcionar melhores resultados. Os resultados apresentados neste artigo foram obtidos por meio de uma simulação, porém, os gestores de Sistemas de Gestão Ambiental estão convidados a utilizarem a avaliação qualitativa paraconsistente e a averiguarem os resultados que vão obter.

\section{Agradecimentos}

Agradeço a Marcos Alberto de Oliveira, auditor de sistemas de Qualidade ISO 9000 Lead Assessor pelo BVQI, pela grande colaboração dada a este artigo. 
ABE, J. M., Fundamentos da lógica anotada, 1992, 139 f.. Tese (Doutorado em Filosofia) - Departamento de Filosofia da Faculdade de Filosofia, Ciências e Letras da Universidade de São Paulo, São Paulo, 1992.

BISPO, C. A. F.; CAZARINI, E. W., A paraconsistent evaluation of software quality according to the McCall factors. In: INTERNATIONAL CONFERENCE ON INDUSTRIAL ENGENEERING AND OPERATIONS MANAGEMENT, 10., Florianópolis, 2004. Proceedings: Knowledge engineering and management applied for the development of productive systems, Porto Alegre: Associação Brasileira de Engenharia de Produção ABEPRO, 2004, p. 227-234. ISBN: 85-88478-13-7.

BUREAU VERITAS QUALITY INTERNATIONAL BVQI., Sistema de Gestão Ambiental. São Paulo: BVQI do Brasil Sociedade Certificadora Ltda., 2004. 13 p.

COSTA, N. C. A.; ABE, J. M.; SUBRAHMANIAM, V. S., Remarks on annotated logic. Zeitschrift für Mathematische Logik und Grundlagen der Mathematik, Berlin, v. 37, p. 561-570, 1991.
COSTA, N. C. A.; ABE, J. M.; SILVA, F. J. I.; MUROLO, A. C.; LEITE, C. F. S., Lógica paraconsistente aplicada. São Paulo: Atlas, 1999. 214 p. ISBN: 85-224-2218-4.

DRUCKER, P. F., O gerente eficaz. 7. ed. Rio de Janeiro: Zahar, 1978. $185 \mathrm{p}$.

MAIMOM, D., ISO 14001: passo a passo da implantação nas pequenas e médias empresas. Rio de Janeiro: Qualitymark Editora, 1999. 86 p. ISBN: 85-7303-214-6.

NEVES, J. L., Pesquisa qualitativa: características, usos e possibilidades. Cadernos de pesquisas em administração, São Paulo, v. 1, n. 3, p. 103-113, 2 sem. 1996.

NBR ISO 14001: 2004. Sistema de gestão ambiental - especificação e diretrizes para uso. Rio de Janeiro: Associação Brasileira de Normas Técnicas, 2004. 14 p.

OLIVEIRA, M. A. de., Em busca da excelência empresarial. São Paulo: DVS Editora, 2004. 124 p. ISBN: 8588329-12-3.

POPE, C.; MAYS, N., Reaching the parts other methods cannot reach: an introduction to qualitative methods in health and health service research. British Medical Journal, London, n. 311, p. 42-45, 1995.

\title{
PARACONSISTENT QUALITATIVE EVALUATION OF AN ENVIRONMENTAL MANAGEMENT SYSTEM IMPLEMENTATION PROCESS
}

\begin{abstract}
A proposal is presented for a new process of qualitative evaluation of the implementation of an Environmental Management System (EMS) based on the ISO 14001: 2004 standard, with a view to its future ISO certification. The proposed evaluation applies the resources of Two-Value Annotated Paraconsistent Logic (2VAPL). This article describes how this evaluation is carried out, since it differs from other qualitative evaluations, and explains its importance for the area of Environmental Management. A comparison made between the results from a simulation of a traditional qualitative evaluation and those obtained from a paraconsistent qualitative evaluation. Also discussed are the tools employed to illustrate the scenario involving paraconsistent analyses, i.e., the Para-Analyzer Algorithm and the Unitary Picture of the Cartesian Plane.
\end{abstract}

Keywords: environmental management system, paraconsistent qualitative evaluation, two-value annotated paraconsistent logic. 
